\title{
Radiation Shielding Issues for MuCool Test Area at Fermilab
}

\author{
I. Rakhno, C. Johnstone \\ Fermilab, P.O. Box 500, Batavia, IL 60510
}

May 2, 2005

\begin{abstract}
The MuCool Test Area (MTA) is an intense primary beam facility derived directly from the Fermilab Linac to test heat deposition and other technical concerns associated with the liquid hydrogen targets being developed for cooling intense muon beams. In this study the origin of the outgoing collimated neutron beam is examined. An alternative shielding option for MTA is investigated as well as the hypothetical worst case of experimental setup is considered.
\end{abstract}

\section{Introduction}

The MTA facility is being designed to test targets and other muon cooling apparatus using the intense Fermilab Linac beam [1]. A detailed radiation shielding study for the area around MTA was performed previously [2] with the MARS14 [3] code. Since then a request along with additional issues have been addressed. First, a request was issued to remove the required shielding block in the refrigerator room and replace it with a block in the target hall near the penetrations. In such a case the helium transfer lines would have a bend as large as $45^{\circ}$ inside the shield block itself. Second, radiation safety regulations require that the radiation environment be estimated for the "worst case" regarding the experimental setup. The issues are addressed in the study here which was performed using the MARS14 code for normal operation. The calculations were performed with the MCNP option turned 'on' to provide the most accurate treatment of low-energy neutron transport [4] presently available.

The requested intensity of the proton beam for the MTA is essentially the full Linac capability [1], or $1.3 \times 10^{13}$ protons per pulse at a $15 \mathrm{~Hz}$ repetition rate and an energy of $400 \mathrm{MeV}$. This intensity represented in this study is a factor of two beyond the current safety envelope of Fermilab Linac. If it is later determined the safety envelope cannot be practically increased, the reduced intensity is still considered acceptable and sufficient to test the MuCool targets and apparatus. Thus, the beam intensity remains $2 \times 10^{14}$ protons per second in all calculations unless otherwise stated. 


\section{The Origin of the Outgoing Collimated Neutron Beam}

In our previous study [2] it was shown that a collimated neutron beam is formed by the existing penetrations to the service building and the beam is directed toward a parking lot. There was a controversy about the origin of the beam because such a collimation is usually associated with forward direction, i.e. along the beam line itself. However, from physical standpoint there is no obstacle for collimation of secondary particles generated in a target in elastic and inelastic nuclear collisions and emitted at large angles. The difference between the latter case and collimation in forward direction is mostly in beam intensity.

To clarify the problem, a separate calculation has been performed for the same system with the only difference - the processes of elastic scattering in the bulk shielding between the target hall and service building were artificially turned OFF, i.e. the elastic scattering cross section for all the nuclei in the shielding was assumed to be zero. The calculated dose distribution for such a case is shown in Fig. 1. A comparison with the realistic dose distribution calculated with realistic elastic scattering cross sections and shown in the same Figure reveals that the turning elastic scattering OFF reduces the dose at the downstream entrance to the penetrations in the refrigerator room within a factor of 100 . Thus the comparison confirms our initial suggestion that the bulk shielding serves as a regular collimator and generates the collimated beam due to small-angle scattering from the walls inside the penetrations.
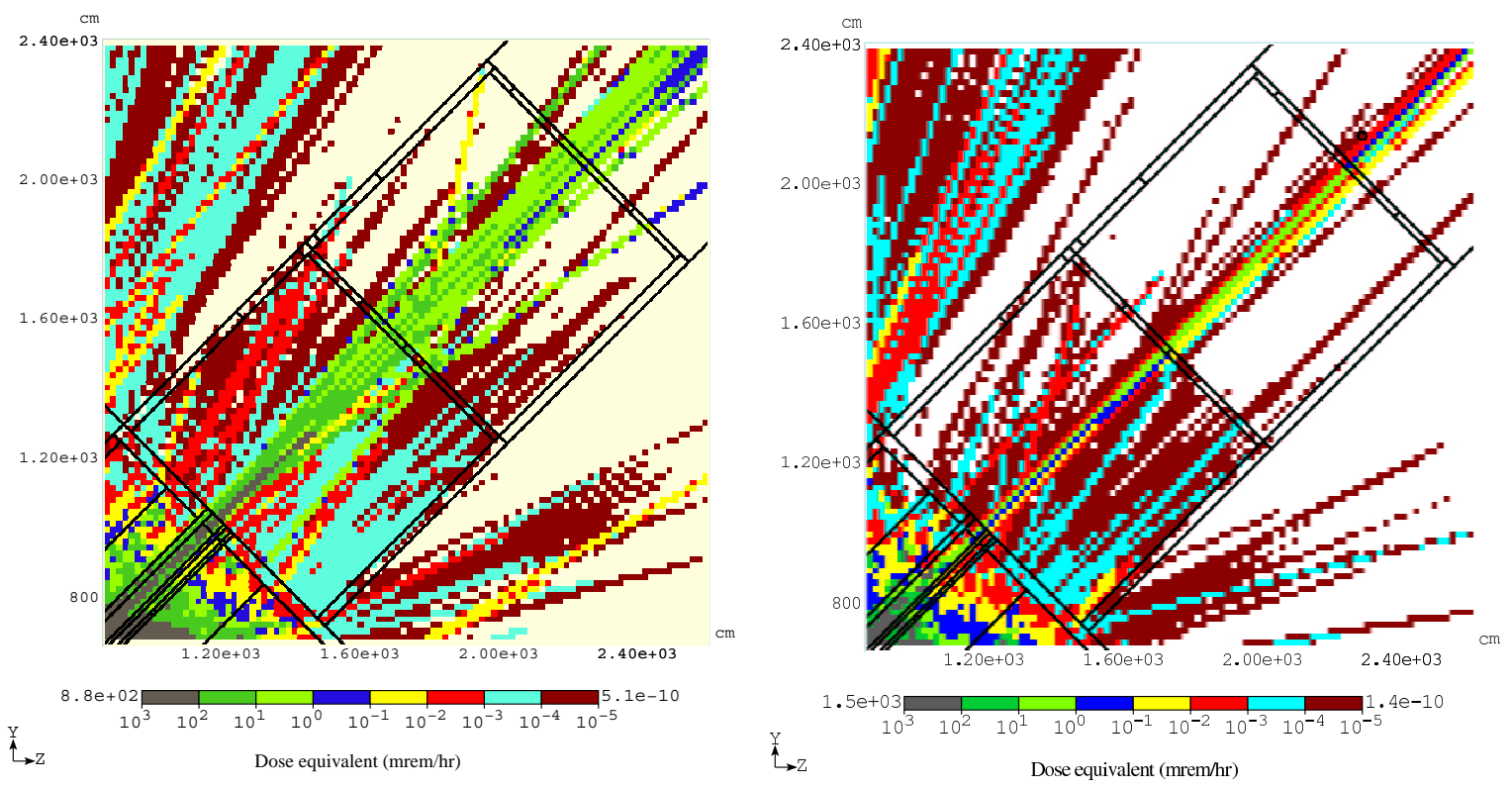

Figure 1: Calculated dose distributions in the service building of the MTA with elastic scattering in the shielding turned ON (left) and OFF (right). 


\section{An Alternative Shielding Option}

In our previous study [2] it was shown that a shielding block is required in the refrigerator room to absorb the collimated neutron beam formed by the existing penetrations and directed toward a parking lot. Although the block occupies less than $2 \%$ of total area of the room, it was considered as the least preferable option from operational standpoint. Therefore, another shielding option was proposed for consideration, namely a concrete block $(-185 \mathrm{~cm} \leq X \leq 215 \mathrm{~cm}, 245 \mathrm{~cm} \leq Y \leq 345 \mathrm{~cm}, 380 \mathrm{~cm} \leq Z \leq 580 \mathrm{~cm})$ at the upstream entrance to the penetrations in the target hall as shown in Fig. 2. The origin of the employed Cartesian coordinate system, $(0,0,0)$, is chosen at the geometrical center of the target.

As for the color scheme employed to denote materials in the geometry model, the following convention applies to any system: white, black, light blue, green, and grey colors correspond to vacuum, black hole (artificial material used in MARS modeling that absorbs $100 \%$ of incoming radiation), air, soil, and regular concrete, respectively. (The meaning of the other colors can vary depending on materials used in the system under consideration.) It should be taken into account also that boundaries between different regions are shown with black lines. When the resolution of the figure is inadequate, small regions sometimes are not distinguishable and appear as black regions.
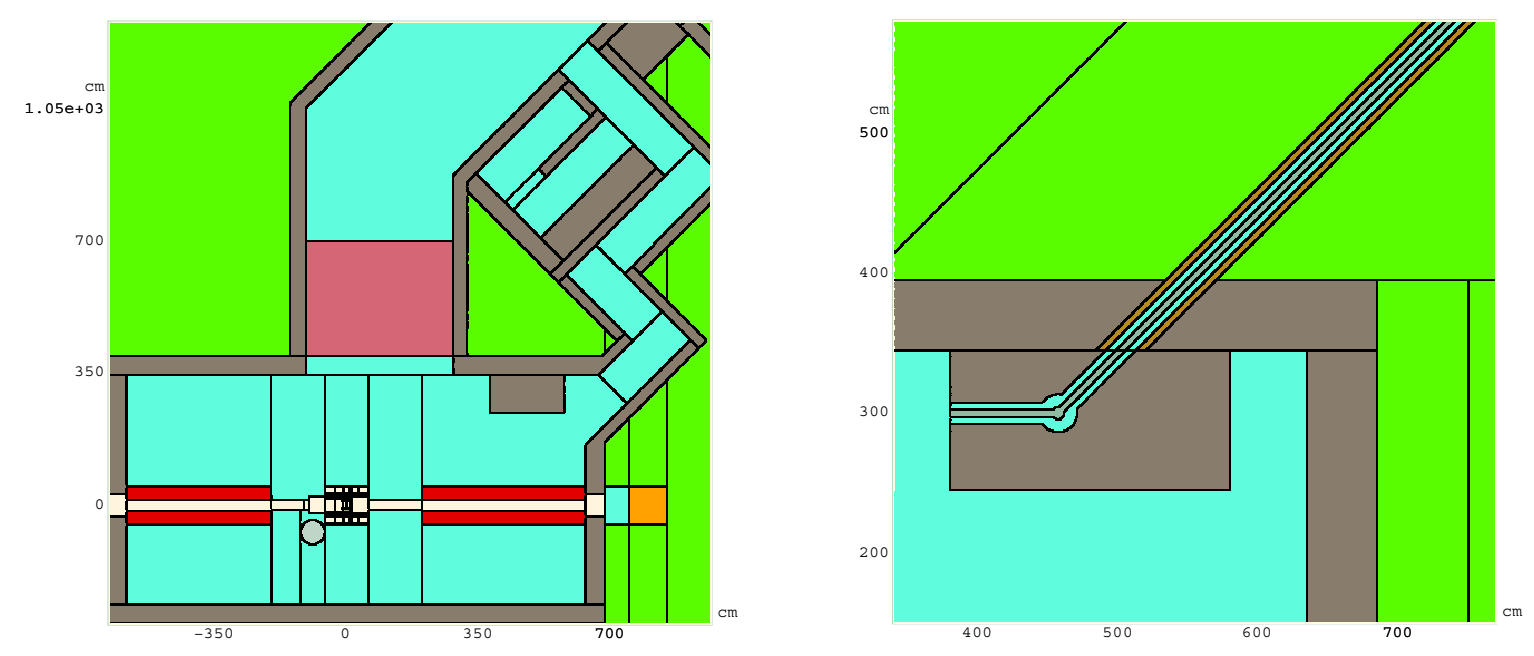

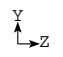

$\stackrel{\mathrm{Y}}{\leftrightarrow} \rightarrow \mathrm{Z}$

Figure 2: Plan view of the MTA with the concrete shielding block $(4 \mathrm{~m} \times 1 \mathrm{~m} \times 2 \mathrm{~m})$ near the penetrations in the target hall at the labyrinth level (left) and the block at the level of the $10^{\prime \prime}$ penetration (right). The helium transfer line and $2^{\prime \prime}$ thick sand filling are shown inside the penetration by means of the light turquoise and beige color, respectively.

The helium transfer line must bend at $45^{\circ}$ inside the shielding block to reduce the probability of neutron streaming through the penetrations. Even with the labyrinth bend in the transfer line, the largest penetration to the equipment rooms still requires further shielding. Therefore, it was assumed in the model that the sand as shown in Fig. 2 fills the 
$10^{\prime \prime}$ penetration; all the other penetrations at different elevations, one $8^{\prime \prime}$ in diameter and four $4^{\prime \prime}$ in diameter, were considered filled with air. The results of calculations, performed for a $1 \mathrm{~cm}$ thick copper disk as a target [2], are shown in Fig. 3. One can see from the dose distribution that the number of neutrons streaming through the penetrations is significantly attenuated when compared to the initial case without the block in target hall (see Fig. 9 in [2]). In current model the dose in the parking lot is approximately $0.1 \mathrm{mrem} / \mathrm{hr}$. If one takes into account the additional factor of two mentioned above, consistent with the current safety envelope of Fermilab Linac, then the dose would be $0.05 \mathrm{mrem} / \mathrm{hr}$ which corresponds to an area of unlimited occupancy [5]. Since there are uncertainties associated with the twodimensional histogram employed to represent the dose distribution, the statistical nature of the Monte Carlo calculations, and future experiments in the MTA, any increase in the dose rate can likely be handled with a removable local shielding near door in the compressor room (as originally proposed) or outside the service building.

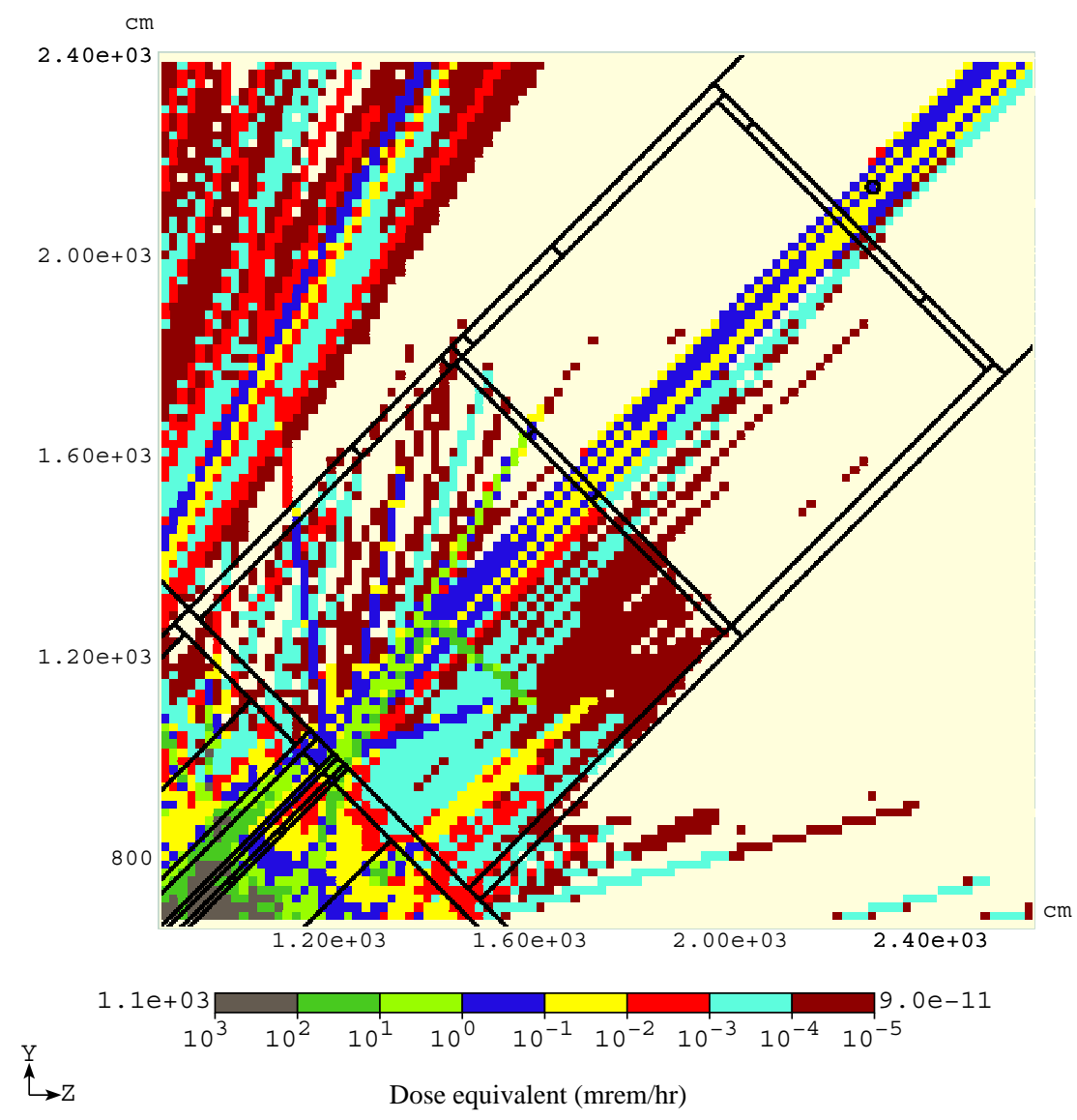

Figure 3: Calculated dose distribution in the service building of the MTA with the shielding block in the target hall (see text). 


\section{The Hypothetical Worst Case}

The radiation safety requirements impose a requirement to perform an estimate for the worst radiation environment that can be achieved within potential, even hypothetical, experimental configurations (see Ref. [5], Article 812). As far as the MTA is concerned, it is assumed in our study that the target has the only degree of freedom - along the $Z$-axis (see Fig. 2) since the beamline is fixed in transverse position. For this case, assuming uniform longitudinal shielding above the target hall, one can neglect the dose variations atop the berm $v s$ the target position. Only the dose variation in the most sensitive area around the MTA, i.e. in the parking lot, requires study.

When considering the collimated neutron beam formed by the penetrations between the target hall and service building and ultimately delivered to the parking lot [2], one can see that the worst case is implemented when the secondary neutrons, generated in inelastic collisions in the target, strike the shielding block containing the helium transfer line at the smallest achievable incident angle (see Fig. 2). Clearly, the smallest incident angle is achieved when the target is moved as far upstream as possible, at the entrance from the pre-cast tunnel to the experimental hall. This is the configuration model for the worst-case experimental setup as used in this study and results for calculations performed for a $1 \mathrm{~cm}$ thick copper disk as a target [2], are shown in Fig. 4. For the hottest spots in the parking lot the predicted prompt dose is about $1 \mathrm{mrem} / \mathrm{hr}$ which implies a tenfold increase when compared with the presently-proposed setup (see Figs. 2 and 3). One must take into account all the relevant uncertainties mentioned in the previous section. The observed increase in dose, however, can be effectively compensated with local shielding if an experiment requires this particular target and location.
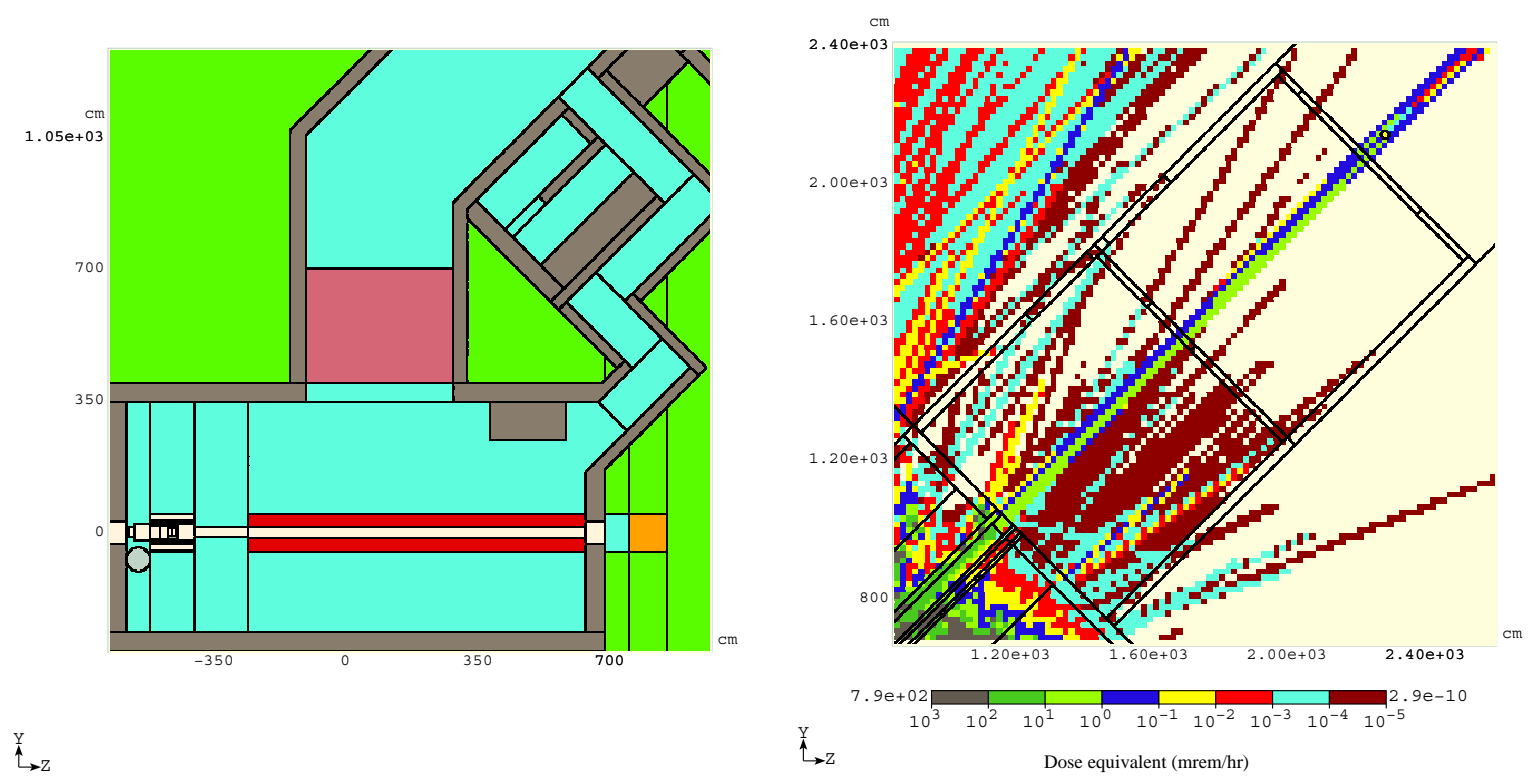

Figure 4: The worst case experimental setup in the MTA: a MARS model (left) and calculated dose distribution in the service building and parking lot (right). 


\section{Conclusions}

The alternative shielding option with the block in the MTA target hall near the penetrations provides for an adequate shielding to classify the parking lot near the booster tower as area of unlimited occupancy (dose rate not exceeding $0.05 \mathrm{mrem} / \mathrm{hr}$ ) at the beam intensity up to $10^{14} \mathrm{p} / \mathrm{s}$ and for a target as thick as $10 \%$ of proton interaction length. Another shielding option is also feasible - moving the door between the refrigerator and compressor rooms to the left or right and putting extra shielding at the location of the old doorway which is directly in the path of the beam.

The worst-case general scenario gives rise to a tenfold increase in the dose rate at the parking lot, other things being equal. Such an experimental target configuration will require either local shielding in the target hall or an additional shielding block in the service building or outside. Alternatively operation at a reduced beam intensity (repetition rate) can be implemented with appropriate safety measures to reduce the dose. (The appropriate, critical-device pulsed-power supplies can be hardwired to run at a reduced repetition rate below the $15 \mathrm{~Hz}$ capability of the Linac.)

\section{Acknowledgements}

The authors are thankful to Alan Bross, Don Cossairt, Michael Gerardi, Bill Higgins, Kamran Vaziri, and Nikolai Mokhov of Fermilab for helpful discussions.

The work was supported by the Illinois Board of Higher Education with the Higher Education Cooperative Act Grant and Universities Research Association, Inc., under contract DE-AC02-76CH03000 with the U. S. Department of Energy.

\section{References}

[1] http://www.fnal.gov/projects/muon_collider/cool/cool.html; D. Errede, R. Alber, A. Bross et al. Proc. of the 2003 Part. Accel. Conf., Portland, OR, USA, May 2003.

[2] I. Rakhno, C. Johnstone, "Radiation Shielding Calculations for MuCool Test Area at Fermilab", Fermilab-TM-2248 (2004).

[3] N.V. Mokhov, “The MARS Code System User's Guide”, Fermilab-FN-628 (1995); N. V. Mokhov, O. E. Krivosheev, "MARS Code Status", Proc. of the Monte Carlo 2000 Conference, Lisbon, October 23-26, 2000, Springer, p. 943; Fermilab-Conf-00/181 (2000); http://www-ap.fnal.gov/MARS/

[4] J.F. Briesmeister, editor, "MCNP - A General Monte Carlo N-Particle Transport Code", Version 4C. Pub. LA-13709-M, Los-Alamos National Laboratory (2000).

[5] "Fermilab Radiological Control Manual", Article 236, http://www-esh.fnal.gov/FRCM/. 